\title{
СОВРЕМЕННЫЕ ВЗГЛЯДЫ НА ЭТИОПАТОГЕНЕЗ, ДИАГНОСТИКУ И ТЕРАПИЮ ПАРАДЕНТАЛЬНОЙ КИСТЫ (ЛИТЕРАТУРНЫЙ ОБЗОР)
}

\author{
MODERN VIEWS ON ETIOPATHOGENESIS, \\ DIAGNOSIS AND THERAPY \\ OF PARADENTAL CYST \\ (LITERARY REVIEW)
}

\section{A. Zhevlakov I. Borodulina}

Summary. The literature review examines the prevalence of a paradental cyst, its etiology and pathogenesis in difficult eruption of the lower third molar, its retention and dystopia. Questions of differential diagnostics, features of clinical course and justification of tactics of pathogenetic therapy are described. The study was conducted based on the search and study of original articles on the etiopathogenesis, diagnosis and treatment of odontogenic cysts, paradental cysts with difficult wisdom tooth eruption in databases: Russian state library, elibrary, PubMed, the Cochrane Library, Google Scholar. The selection of literary sources was carried out by keywords.

Keywords: odontogenic cysts, paradental cyst, lower third molar, wisdom tooth, wisdom tooth retention, wisdom tooth dystopia, wisdom tooth extraction.

\section{Вве $а$ ение}

D азнообразие концепций и подходов к анализу этиопатогенеза парадентальной кисты обуславливает повышенный интерес к поиску высокоинформативных методов диагностики и обоснования патогенетической терапии.

\section{Цель работы}

Анализ современных взглядов на вопросы этиологии и патогенеза парадентальной кисты, особенностей клинического течения, методов диагностики и патогенетической терапии.

\section{Материалы и метолы}

Исследование проводили на основе поиска и изучения оригинальных статей по вопросам этиопатогенеза, диагностики и лечения одонтогенных кист, парадентальных кист при затрудненном прорезывании зуба мудрости в базах данных: Российская государственная библиотека, eLibrary, PubMed, The Cochrane Library, Google
Жевлаков Андрей Андреевич

Аспирант, ФГБ ВОУ ВО «Военно-медицинская академия имени С. М. Кирова», Санкт-Петербург dr.zhevlakovandrey@mail.ru

Бородулина Ирина Ивановна

Д.м.н., профессор, ФГБ ВОУ ВО «Военно-медицинская академия имени С. М. Кирова», Санкт-Петербург borodulina59@mail.ru

Аннотация. В литературном обзоре рассматриваются вопросы распространенности парадентальной кисты, этиологии и патогенеза её формирования при затрудненном прорезывании нижнего третьего моляра, его ретенции и дистопии. Описаны вопросы дифференциальной диагностики, особенностей клинического течения и обоснования тактики патогенетической терапии. Исследование проводили на основе поиска и изучения оригинальных статей по вопросам этиопатогенеза, диагностики и лечения одонтогенных кист, парадентальных кист при затрудненном прорезывании зуба мудрости в базах данных: Российская государственная библиотека, elibrary, PubMed, The Cochrane Library, Google Scholar. Отбор литературных источников осуществлялся по ключевым словам.

Ключевые слова: одонтогенные кисты, парадентальная киста, нижний третий моляр, зуб мудрости, ретенция зуба мудрости, дистопия зуба мудрости, удаление зуба мудрости.

Scholar. Отбор литературных источников осуществлялся по ключевым словам.

\section{Результаты исслеАования}

Парадентальная киста до недавнего времени считалась достаточно редким заболеванием, и впервые была выделена ВОЗ в качестве самостоятельной нозологической формы только в 1992 году. Это было обусловлено трудностями дифференциальной диагностики, когда ее рассматривали либо как «перикоронарит», либо как «боковую пародонтальную кисту», либо любое другое заболевание, обусловленное воспалением зубного фолликула [1]. Поэтому распространенность парадентальных кист считалась достаточно низкой по сравнению с другими кистами. Так, по данным одних авторов распространенность парадентальных кист составляла 3-5\% от числа всех одонтогенных кист [2]. Данные других авторов подтверждали эти сведения: парадентальные кисты составляли 4,7\% из 1051 одонтогенных кист [3]. Имеются сведения о частоте парадентальных кист в диапазоне от 0,9 до 4,7\% от общего числа одонтогенных кист [4]. 
Испанскими учеными была изучена частота распространенности различных типов одонтогенных кист путем проведения ретроспективного анализа 418 случаев одонтогенных кист у 380 пациентов, включенных в базу данных Центра хирургической стоматологии и имплантологии Барселонского университета за 10 лет. Было установлено, что чаще всего одонтогенные кисты встречались у мужчин (58.4\%), средний возраст которых составил 42 года, с диапазоном от 7 до 83 лет. Самыми частыми диагнозами были «радикулярная киста» $(50,2 \%)$ и «фолликулярная киста» $(21,8 \%)$. Парадентальная киста среди одонтогенных кист по распространенности занимала 3-е место (20,8\%) [5]. Чаще всего парадентальные кисты встречались у мужчин после 30 лет, хотя были зарегистрированы случаи и у детей [6]. Заслуживает внимания и тот факт, что распространенность разных типов одонтогенных кист в динамике 10-летнего ретроспективного исследования имела разнонаправленные характеристики: показатель распространенности фолликулярных кист снижался, а радикулярных и парадентальных кист увеличивался [7].

Недавними исследованиями японских ученых было показано, что самая высокая частота одонтогенных кист отмечена в возрасте 30 лет. Несмотря на это, средний возраст пациентов составлял 46,0 \pm 13,4 лет. По-видимому, это было обусловлено высокой распространенностью данной патологии у лиц, намного старше 40 лет и у лиц в возрасте 20 лет и моложе [8]. Что касается гендерного распределения, то многие клинические наблюдения отмечают явную предрасположенность у мужчин к развитию одонтогенных кист [9].

В отечественной литературе вопросы этиопатогенеза, клиники и диагностики парадентальных кист освещены недостаточно и публикации о них носят единичный характер. Так, если происхождение радикулярных одонтогенных кист связано с хроническим воспалением в периодонте, происхождение фолликулярных кист объясняется нарушением развития зубного фолликула, то возникновение парадентальных кист до конца не выяснено [10]. До сих пор существуют различные, порой противоречивые, взгляды по вопросам этиологии и патогенеза данного заболевания. В этой связи непонятно, что является пусковым механизмом происхождения парадентальной кисты [11].

Зуб мудрости в области формирования парадентальной кисты, является, как правило, жизнеспособным. Обычно на верхней челюсти зуб мудрости имеет один корень, а на нижней челюсти два корня. В литературе имеются сведения, что у представителей азиатского континента нормальным явлением считается наличие трех корней у нижнего зуба мудрости, что объясняется генетической предрасположенностью [12, 13]. Среди генетически обусловленных аномалий зубов мудрости, является микродентия, которая характерна для боковых резцов верхней челюсти и зубов мудрости нижней челюсти [14]. Имеются также литературные данные о том, что зубы мудрости в 86\% случаев на верхней челюсти и в 52,4\% случаев на нижней челюсти имеют слитые воедино корни [15].

Обычным явлением, связанным с затрудненным прорезыванием нижнего зуба мудрости, является его импакция, обусловленная механическим препятствием для его роста со стороны уже прорезывавшегося нижнего второго моляра [16]. В исследованиях, занимавшихся вопросами импакции нижнего зуба мудрости, гендерной предрасположенности выявлено не было [17]. По данным ряда авторов, 70\% импакций характеризуются наличием перикоронарита, повреждением соседнего второго моляра или формированием кисты в области корня нижнего зуба мудрости $[18,19]$.

Патогенез парадентальной кисты при затруднённом прорезывании зуба мудрости часто связывают с возникновением костного кармана в дистальном отделе нижней челюсти, выполненного фиброзной тканью с наличием эпителиальных клеток Малассе. Именно эпителиальные клетки Малассе, происходящие из периодонта интактного зуба мудрости, запускают процесс формирования оболочки, выстланной многослойным плоским эпителием, и развитию кисты прорезывания [20].

Сведения об участии эпителиальных клеток Малассе в этиологии парадентальной кисты впервые появились в литературных источниках в 80-х годах прошлого столетия. Патогенез парадентальной кисты связывали с воспалительным компонентом, являвшимся пусковым механизмом патологической активности эпителиальных клеток Малассе. Происхождение парадентальной кисты рассматривали из редуцированного эпителия эмали в результате одностороннего расширения зубного фолликула на фоне вторичного воспаления периодонта и пародонта [21]. Воспалительное происхождение парадентальной кисты было обусловлено перикоронаритом во время прорезывания зуба мудрости, а наиболее вероятными источниками эпителия кисты, по мнению авторов, являлся редуцированный эмалевый эпителий Малассе [22].

В этиопатогенезе парадентальных кист хроническое воспаление в области нижнего третьего моляра и, в частности, перикоронарит имеет основополагающее значение. Хроническому воспалению могут также способствовать полуразрушенные нижние третьи моляры, провоцируя воспаление под слизистой оболочкой десны [23]. 
Формированию парадентальной кисты может способствовать адгезия пародонтального кармана к эмали и цементу зуба с последующим воспалением [24]. Некоторые авторы описывают адгезию пародонтального кармана к твердым тканям зуба как «спаянно-сулькулярный эпителиальный механизм» происхождения парадентальной кисты [25]. Вследствие последующего воспаления спаянно-сулькулярного участка пародонта формируется парадентальна киста в непосредственной близости к шейке зуба со стороны боковой поверхности корня [26].

A. Mohan et al. (2017) показали, что формированию парадентальной кисты могут способствовать повторный воспалительный процесс в периодонте либо рецидив перикоронарита. Нагноение проникает через пародонтальный карман или зубодесневую щель. Поражается щечно-дистальная, реже мезиальная поверхность частично или полностью прорезывавшегося интактного жизнеспособного зуба мудрости [27].

В литературе встречаются такие синонимы парадентальной кисты, как «киста затрудненного прорезывания зуба мудрости», «ретромолярная киста». Это обусловлено тем, что при затрудненном прорезывании третьего моляра нижней челюсти между челюстной костью и зубом мудрости формируется костный карман, заполненный фиброзной тканью. По мере закрытия сообщения костного кармана с полостью рта, образуется изолированная полость с формированием парадентальной кисты [28].

Причинами затрудненного прорезывания нижних зубов мудрости являются неблагоприятные топографо-анатомические условия, обусловленные недостатком места в нижней челюсти. Гайворонский И.В. и соавт. (2015) провели краниометрические измерения на 250 черепах взрослых людей с целью выявления анатомических причин, способствующих затрудненному прорезывания зубов мудрости. Для этого изучали основные морфометрические характеристики нижней челюсти, а также взаимосвязь этих признаков с основными морфометрическими параметрами лицевого черепа. Установлено, что основной причиной затрудненного прорезывания зуба мудрости является диспропорция размеров его коронковой части и размеров участка тела нижней челюсти для адекватного позиционирования зуба. Комплексные морфометрические исследования черепов показали, что затрудненное прорезывание нижних зубов мудрости чаще встречается у лептопрозопов, чем у мезо- и эурипрозопов, что обусловлено меньшими показателями ретромолярного пространства у лептопрозопов [29].

Размеры челюстей, рост лица и размеры зубов варьируют в зависимости от расовой принадлежности, эт- нической культуры и наследственных факторов и также влияют на процесс прорезывания зубов мудрости [30]. Атипичное расположение зачатка нижнего третьего моляра, патология роста и развития нижней челюсти приводят к аномалии положения сформировавшегося нижнего восьмого зуба (дистопии), а также к задержке процесса прорезывания (ретенции). Неправильное положение зуба мудрости в челюсти нередко способствует развитию воспалительного процесса, длительное существование которого подготавливает почву для возникновения перикоронарита [31, 32].

Особенностям развития перикоронарита при затрудненном прорезывании зуба мудрости посвящено исследование индийских ученых. Авторы установили, что в возрастном аспекте перикоронарит чаще всего ассоциируется с возрастом 20-29 лет, в гендерном аспекте более распространен среди женщин (62,7\%). Авторы объясняют это тем, что у женщин челюсти перестают расти раньше, чем у мужчин, когда нижние зубы мудрости только начинают прорезываться. Замечено также, что беременность провоцирует развитие перикоронарита, особенно во втором триместре. Большое значение авторы уделили влиянию углу наклона зуба мудрости в нижней челюсти на развитие перикоронарита. Дистоангулярный наклон преобладал над другими положениями зуба и составлял 49,5\%, а меньше всего перикоронарит встречался при горизонтальном положении зуба. По мнению авторов, при дистоангулярном наклоне зуба мудрости толстая слизистая ретромолярная подушка подвергается высокому давлению, перфорируется и способствует частичному открытию коронки зуба [33].

Сформировавшийся капюшон заполняется и накапливает пищевые остатки, инфицируется и воспаляется [34]. Если лечение является симптоматическим, заболевание переходит в хроническую стадию с периодическими обострениями. Зубы мудрости, частично покрытые мягкими тканями, больше подвержены периодическому воспалению по сравнению с зубами мудрости, полностью ретенированными в челюстной кости или с полностью прорезывавшейся коронкой зуба [35].

Если дисто-ангулярный наклон ретинированного зуба мудрости способствует развитию перикоронарита, то мезо-ангулярный наклон этого зуба проявляется импакцией соседнего второго моляра. Третий моляр, который частично прорезан и занимает мезо-ангулярное положение, способствует поражению соседнего второго моляра в виде развития кариеса в дистальном отделе шейки зуба $[36,37]$. Установлено, что в 59\% случаев на нижней челюсти и в $38 \%$ случаев на верхней челюсти импакции вызывают повреждение соседних вторых моляров в виде кариеса, резорбции межзубной перегородки или формирования кисты [38, 39]. 
Объективным анатомо-топографическим критерием нормальных размеров нижней челюсти для полноценного прорезывания зуба мудрости является величина «ретромолярного расстояния» (расстояние между дистальным краем коронки второго моляра и нижнечелюстным отверстием соответствующей стороны нижней челюсти). Согласно антропометрическим измерениям, проведенным в 1971 году А. Т. Руденко, физиологическая величина ретромолярного расстояния должна быть равна 29 мм. В челюстях с ретинированными зубами мудрости величина ретромолярного расстояния сокращалась вплоть до 22,4 мм. В специальной литературе физиологическая величина ретромолярного расстояния именуется как «положительная диастема по А.Т. Руденко» [40].

Исследования, проведенные А.К.Иорданишвили и соавт. (2017) с помощью КЛКТ челюстных костей у пациентов с полностью прорезывавшимися зубами мудрости показали, что величина ретромолярного расстояния на нижней челюсти должна составлять не менее 26,5 мм. Расстояние между дистальным краем второго нижнего моляра и передним краем ветви нижней челюсти должно быть не менее 14,5 мм при толщине альвеолярной части нижней челюсти в ретромолярной зоне не менее 13,5 мм. Авторы подчеркивают, что даже при наличии необходимого объема костной ткани в области нижних зубов мудрости для их прорезывания необходимо отсутствие снижения высоты альвеолярной части нижней челюсти [41].

Ряд авторов считают, что возникновению парадентальной кисты во многом способствует положение нижнего зуба мудрости. Так, основными положениями нижних восьмых зубов, при которых формируются парадентальные кисты, явились дистально-косое положение и горизонтальное положение, с расположением части ретинированного зуба в области ветви нижней челюсти [42].

Ученые S. Núñez-Urrutia, R. Figueiredo, C. Gay-Escoda (2010) из Барселонского университета изучали предпочтительные места локализации парадентальных кист. При этом они учитывали пол, возраст, клиническую картину (размер и расположение очага), рентгенологическую картину, продолжительность заболевания, проведенное лечение, наличие осложнений и рецидивов. Было установлено, что размеры поражения парадентальных кист варьировали от 2 до 60 мм, при этом средний размер составил 18,4 мм. Наиболее предпочтительным местом локализации одонтогенных кист являлась нижняя челюсть (61.5\%), а именно область нижнего зуба мудрости (36.8\%). Что касается локализации парадентальных кист, то по результатам представленного исследования, на область нижних третьих моляров приходилось $97,7 \%$.
Этот факт совпадает с данными, свидетельствующими о распространенности данного вида кист в области нижнего третьего моляра в 99,4\% случаев, причем двусторонние локализации отмечены в 23,6\%. На рентгенограмме они имеют форму полумесяца, с четкими ровными краями и наложением на область корней [43].

Однако литературные сведения о распространенности парадентальных кист в области нижнего зуба мудрости не всегда совпадают. Так, по одним данным, парадентальные кисты в области нижних третьих моляров по распространенности занимают 2-е место из всех видов одонтогенных кист (25\%). По другим данным, парадентальные кисты в области нижних третьих моляров располагаются 61,4\% случаев [44].

Парадентальная киста локализуется в основном в области нижней челюсти и, почти всегда, на дистальной или щечной стороне полностью или частично прорезавшегося третьего моляра. Тем не менее, очень редко, но все же имеет место быть задействованными как мезиальная поверхность, так и язычная поверхность нижнего зуба мудрости [45].

Кроме того, имеются сведения о нетипичных местах локализации парадентальных кист, а именно в области нижнего второго премоляра и нижнего первого и второго моляра. Y. Morimoto, T. Tanaka, I. Nishida et al., (2004) рассматривали эти вариации клинического вида кисты как две самостоятельные группы. Первая группа была связана со вторым постоянным нижним премоляром, первым и вторым моляром; вторая группа была связана только с третьим нижним моляром.

В литературе описан редкий клинический случай формирования парадентальной кисты в области нижнего зуба мудрости, слившегося с редуцированным сверхкомплектным моляром. Гистологически было установлено, что слияние зубов произошло на стадии формирования зубных зачатков. Подобные случаи слияния обычно отмечают со стороны молочных зубов во фронтальном отделе челюстей. Этиология и патогенетические механизмы слияния зачатков зубов до конца не изучены. Есть мнение, что давление соседних зубных фолликулов вызывает контакт наиболее близкорасположенных зачатков, что вызывает их плотный контакт с последующей кальцификацией. В данном клиническом случае зуб мудрости располагался горизонтально и сливался с сверхкомплектным зубов. Со стороны сверхкомплектного рудиментированного зуба размещалась парадентальная киста в непосредственной близости от корня зуба мудрости. В анамнезе заболевания у пациента был установлен хронический рецидивирующий перикоронарит, что объясняет связь парадонтального кармана (капюшона) с парадентальной кистой [46]. 
В.Ф.Черныш (2018) считает, что парадентальные кисты по гистологическому строению принципиально не отличаются от других одонтогенных кист; выстланы многослойным плоским неороговевающим эпителием. A.S. Pinto, A. L. Costa, M. C. Pinto et al. (2016), в связи с тем, что гистопатологические данные при рутинном окрашивании гематоксилином и эозином не являются патогномоничными, важное значение для установления окончательного диагноза придают корреляции с клиническими и визуализационными характеристиками.

Японскими учеными S.Maruyama, M. Yamazaki, A. Tatsuya (2015) также показано, что стенки парадентальной кисты выстланы преимущественно незрелой грунуляционной тканью с участками расширенных сосудов и кровоизлияний, а межклеточное пространство заполнено воспалительными инфильтрациями. Тонкие хаотично разбросанные эпителиальные тяжи соединяют участки оболочки со связочным аппаратом периодонта.

M. Terauchi, S. Akiya, J. Kumagai et al. (2019) дифференцируют парадентальную кисту с примордиальной (кератокистой) кистой и боковой кистой пародонта. Примордиальные кисты могут возникать в любой период жизни. Возникновение кератокист не связывают напрямую с формированием коронки, так как они развиваются после полного завершения формирования зуба, окружая его весь целиком. Чаще всего в патологический процесс вовлекается нижний зуб мудрости, который бывает глубоко погружен в костную ткань и имеет, как правило, мезиальный наклон.

Примордиальная киста не имеет при этом какой-либо существенной корреляции между своим размером и возрастом пациента. Вне зависимости от возраста больного, киста, на фоне отсутствия клинических симптомов и болевой симптоматики, может достигать столь больших размеров, что они могут приводить к патологическому перелому нижней челюсти [47].

Есть мнение, что на величину примордиальной кисты влияет глубина погружения ретинированного зуба в челюсти и толщина кости, покрывающей этот зуб. Объясняют это тем, что киста возникает из восстановленного эпителия эмали и растет под внутренним давлением жидкости, расположенной в пространстве между стенкой кисты и коронкой зуба. Из этого легко понять, что примордиальные кисты легко растут вокруг зуба, полностью окруженного костной тканью и, чем толще костная ткань над зубом, тем выше внутреннее давление жидкости внутри кисты [48].

Большой интерес представляет величина ангуляции ретенированного зуба, погруженного в кисту. Величина ангуляции зуба определялся углом, образованным между пересекающимися продольными осями второго и третьего моляров. Наибольшую частоту встречаемости ангуляции имело горизонтальное положение зуба $(36,3 \%)$, за ним следовали инверсия $(32,5 \%)$ и мезо-угловое положения (25,4\%). Существует вероятность того, что инверсия (перевернутое положение) третьих моляров нижней челюсти, связанное с зубочелюстной кистой, является следствием изменения положения зуба по мере увеличения размеров кисты [49].

B. R. Chrcanovic, R.S. Gomez (2019) в своем исследовании описывают редкую форму примордиальной кисты, которую иногда называют боковой кистой пародонта (боковая периодонтальная киста, латеральная периодонтальная киста), прилежащей к корню зуба и не имеющей признаков воспаления. Латеральная периодонтальная киста имеет сходные клинические и гистоморфометрические признаки с десневой кистой. Данные виды кист имеют похожие места локализации, а именно, переднее расположение: поражения в области резцов и клыков; расположение в области премоляров; заднее расположение: поражения в области моляров и ретромолярном участке. Латеральная периодонтальная киста может сопровождаться повреждениями подлежащей кортикальной пластинки с ее эрозиями и перфорациями, расшатыванием зуба. На рентгенограмме резорбция костной ткани может сочетаться с резорбцией корня по всей его протяженности [50].

Клиника парадентальных кист не имеет характерных специфических признаков. Поэтому из-за сложностей в диагностике парадентальные кисты распознаются не всегда, и часто принимаются за фоллликулярные кисты с соответствующим им лечением. Парадентальная киста, как правило, возникающая в области непрорезавшегося полуретенироваанного нижнего зуба мудрости, растет бессимптомно и, поэтому выявить её в начальной стадии развития бывает очень сложно [51, 52].

Нередко кисту обнаруживают случайно на ортопантограммах при проведении общесанационных стоматологических мероприятий или при обследовании по поводу затрудненного прорезывания зуба мудрости. По мере роста парадентальной кисты в ретромолярной области полуретенированного зуба мудрости появляется локальная безболезненная припухлость. Исследования, проведенные с помощью электроодонтометрии, подтверждают жизнеспособность зуба (от 2 до 6 мкА) [53].

На рентгенограммах выявляется разряжение костной ткани позади непрорезавшегося зуба мудрости округлой или овальной формы с четкими границами в виде полоски остеосклероза, расположенное в области угла или ветви нижней челюсти. Примечательным является 
тот факт, что в области верхушки полуретенированного зуба мудрости сохраняется периодонтальная щель, а сама парадентальная киста располагается возле зуба и охватывает только часть его коронки; расширения периодонтальной щели не наблюдается на всем протяжении непораженной части корня зуба [54].

Рентгенологическое исследование играет важную роль в диагностике, определяет размер поражения и отношение к смежным анатомическм структурам. Однако на рентгенограммах парадентальная киста часто неправильно интерпретируется, так как она может иметь атипичными клинические и рентгенологические особенности [55]. Для повышения информативности и достоверности диагностики большое значение имеют конусно-лучевая компьютерная томография (КЛКТ) и магнитно-резонансная томография (МРТ) [56, 57]. КТ-изображения дают трехмерные изображения костных структур, улучшают контрастирование тканей и обеспечивают лучшее разграничение границ поражения и точных измерений кисты. Однако они не позволяют визуализировать содержимое очага поражения $[58,59]$.

Анализ содержимого очага поражения должен быть обязательным для отличия от других одонтогенных поражений. Такую возможность представляет МРТ-сканирование кисты с низкой и средней интенсивностью сигнала на Т1-взвешенных изображениях и высокой интенсивностью сигнала на Т2-взвешенных изображениях, что согласуется с высокой концентрацией белка и смешанным воспалительным клеточным инфильтратом. В связи с этим МРТ-исследование может предоставить дополнительную информацию относительно жидкого компонента поражения [60].

Операция удаления ретенированного нижнего зуба мудрости является довольно сложной хирургической процедурой, обусловленной особенностями анатомо-топографических характеристик удаляемого зуба. Сюда относятся большое разнообразие формы зубов мудрости, количества и формы их корней, патологических изменений в окружающих тканях (образование парадентальных кист). Традиционная методика удаления ретинированного зуба мудрости по Г.Д.Житницкому сопровождается значительным объемом повреждений костной ткани и мягких тканей. Постэкстракционная рана заживает вторичным натяжением, а воспалительные осложнения в послеоперационном периоде составляют от $14 \%$ до $35 \%[61,62]$.

В современной литературе встречается неоднозначное отношение хирургов к вопросу использования остеорепаративных средств, используемых для заполнения постэкстракционной костной раны. Одни авторы предпочитают тактику ведения раны под кровяным сгуст- ком и йодоформной турундой $[63,64]$. Другие авторы используют для замещения дефекта различные смеси биоматериалов $[65,66]$. Дискуссионным остается вопрос о необходимости использования биорезорбируемых мембран, как факторов оптимизирующих процессы заживления слизистой оболочки над костным дефектом. В этой связи проведение исследований по определению эффективности использования остеопластических средств нового поколения, а также биорезорбируемых мембран при удалении ретенированных нижних третьих моляров являются весьма актуальными.

Для оценки влияния остеопластического материала «Коллост» на регенерацию костной ткани и тонкой коллагеновой биорезорбируемой мембраны «Коллост» на заживление слизистой оболочки были проведены экспериментальные исследования на животных. Полученные данные показали высокий уровень интенсивности остеорепаративного процесса в костной ране, заполненной препаратом «Коллост» в сочетании с биорезорбируемой мембраной «Коллост»; данные рентгенологического исследования и эхоостеометрии указывали на более интенсивные процессы костеобразования в области удаленных зубов. Применение препарата «Коллост» В сочетании с биорезорбируемой мембраной «Коллост» при удалении ретенированных и полуретенированных третьих нижних моляров позволило снизить количество послеоперационных осложнений на 41,9\% по сравнению с пациентами, где заживление раны происходило по традиционной методике [67].

Оостеопластические материалы должны обладать остеоиндуктивностью, биорезистентностью, биосовместимостью, а также способностью выполнять и поддерживать объём дефекта костной ткани. Большое клиническое применение получили композиции гидроксиапатита с коллагеном, способствующие пролиферации фибробластов, васкуляризации близлежащих тканей и формированию новой костной ткани с последующей её перестройкой. Основой для проведения метода тканевой инженерии является присутствие субстрата-носителя и наличие биореакторов, к которым относятся мезенхимальные и стромальные клетки, обогащённая тромбоцитами плазма (ОТП) [68]. Применение ОТП для ускорения роста кости и мягких тканей в настоящее время стало одним из наиболее востребованных направлений в челюстно-лицевой хирургии. ОТП способствует увеличению остеогенной активности и оптимизации процессов репаративного остео- и хондрогенеза. Метод костной пластики при хирургическом лечении ретинированных дистопированных нижних третьих моляров с использованием комбинации остеопластического материала «Стимул-ОСС» и обогащённой тромбоцитами аутоплазмы является по мнению авторов патогенетически обоснованным [69]. 
Т.Л. Маругина и соавт. (2012) с целью поиска альтернативного способа цистэктомии парадентальных кист, сопровождающих болезни прорезывания нижних восьмых зубов провели сравнительный анализ двух оперативных методов лечения: традиционного способа и метода пьезохирургии. При применении метода компакт-остеотомия над ретенированным зубом осуществлялась при помощи ультразвукового аппарата Piezon Master Surgery, далее энуклеация зуба и цистэктомия с последующим удалением зоны склероза. Удаление зоны склероза при помощи пьезохирургии снижало степень ожога костной ткани и способствует уменьшение коллатерального отека, заживлению раны первичным натяжением и снижению сроков временной нетрудоспособности пациента. Ближайшие результаты характеризовались отсутствием выраженного коллатерального отека в $100 \%$ случаев; заживление раны осуществлялось первичным натяжением в 100\% случаев. Данный результат авторы связывают с принципом рабаты ультразвукового аппарата, не генерируют большого количества тепла, и, тем самым, оказывает менее выраженное повреждающее действие на ткани [70].

\section{Выво $\triangle$}

Проведенный обзор литературных источников по вопросам этиопатогенеза, диагностики и терапии парадентальных кист показал актуальность избранного исследования, требующего дальнейших глубоких научных изысканий и практических наблюдений.

Авторы заявляют об отсутствии конфликта интересов.

\section{ЛИТЕРАТУРА}

1. Paradental Cyst (Inflammatory Collateral Cyst): A True Clinicopathologic Entity / R. Rajendran, H. Pillai, K. A. Fouzan, A. Sukumaran // Oral and Maxillofacial Pathology Journal._2015 July-December.—6 (2).—P. 621-624.—URL: https: // www.researchgate.net/publication/279755611

2. Magnusson B. The paradental cyst a clinicopathologic study of 26 cases. / B. Magnusson, H. Borrman // Swed Dent J.— 1995.— 19 (1). — P. 17.

3. Craig G. T. The paradental cyst: a specific inflammatory odontogenic cyst // Br Dent J.— 1976 Jul. — 141(1). — P. 914.

4. The inflammatory paradental cyst: a critical review of 342 cases from a literature survey, including 17 new cases from the author's files. / H.P. Philipsen, P. A. Reichart, I. Ogawa [et al.] // J Oral Pathol Med. — 2004. — 33. - P. 147-55.

5. Núñez-Urrutia S. Retrospective clinicopathological study of 418 odontogenic cysts / S. Núñez-Urrutia, R. Figueiredo, C. Gay-Escoda. — D0I: 10.4317 / medoral.15.e 767 // Med Oral Patol Oral Cir Bucal._ 2010 Sep. - 1. - 15 (5). - P. 767-73.

6. Paradental cyst is an inclusion cyst of the junctional/sulcular epithelium of the gingiva: histopathologic and immunohistochemical confirmation for its pathogenesis / S. Maruyama, M. Yamazaki, A. Tatsuya // Oral Surg Oral Med Oral Pathol Oral Radiol. — 2015 Aug. — 28. - 120(2). — P. 227-237.

7. Chrcanovic B. R. Paradental (mandibular inflammatory buccal) cyst / B. R. Chrcanovic, B. M. Reis, B. Freire-Maia // Head Neck Pathol.— 2011 Jun.- - 5(2). P. $159-164$.

8. An Analysis of Dentigerous Cysts Developed around a Mandibular Third Molarby Panoramic Radiographs / M. Terauchi, S. Akiya, J. Kumagai [et al.]. — D0I: 10.3390/ dj7010013 // Dent. J._ 2019. — 7.— 13.—URL: https://doi.org/10.3390/dj7010013

9. A clinicopathological study of 338 dentigerous cysts / H. P. Lin, Y. P. Wang, H. M. Chen [et al.] // J. Oral Pathol. Med. — 2013. — 42. — P. $462-467$.

10. Cysts and Pseudocysts of the Oral Cavity: Revision of the Literature and a New Proposed Classification / D. Menditti, L. Laino, M. DI Domenico [et al.]. - D0l: 10.21873/inviv0.11340. // In Vivo. — 2018. — Vol. 32 (5). — P. 999-1007. URL: https://www.ncbi.nlm.nih.gov/pmc/articles/PMC6199599/

11. Пономарёв А. А. Закономерности строения верхней и нижней челюстей в норме, при различных видах ретенции и отсутствии зубов мудрости: специальность 14.03.01 «Анатомия человека», 14.01 .14 «Стоматология»: автореферат диссертации на соискание ученой степени доктора медицинских наук / Пономарёв Александр Анатольевич. — Санкт-Петербург, 2018. - 40 с. — Место защиты: Военно-медицинская академия им. С. М. Кирова.

12. Singh S. Root canal morphology of South Asian Indian maxillary molar teeth / S. Singh, M. Pawar // Eur J Dent. — 2015. — 9. — 19. — P. 133-144.

13. Genetic, environmental and epigenetic influences on variation in human tooth number, size and shape / G. Townsend, M. Bockmann, T. Hughes, A Brook // Odontology. - 2012. - 100.-P. 1-9.

14. Sharma A. Concomitant hypodontia and unusual dental anomalies in families / A. Sharma, S. Sharma, V. P. Singh // Journal of Health Specialties. — 2014. - 2. P. 82-86.

15. Ross I. F. Root fusion in molars: incidence and sex linkage / I. F. Ross, P. A. Evanchik // J Periodontol. — 1981. — 52.— P. 663-667.

16. 3rd Molar variations via radiograph / J. Ghazfa, S. Maha, B. Hasan [et al.].— D0l: 10.15761/BEM.1000121 // Biol Eng Med.— 2017.—Vol. 2(3).—P. 1-6.

17. Incidence of impacted mandibular third molars in population of Bosnia and Herzegovina: a retrospective radiographic study / S. Secic, S. Prohic, S. Komsic, A. Vukovic // Journal of Health Sciences. - 2013. - 3. - P. 211-216.

18. The Prevalence Rate of Third Molar Impaction among Jeddah Population / A. M. Bayoumi, R. Baabdullah, A. F. Bokhari, M. Nadershah // Int J Dent Oral Health. 2016. - 2.- P. 10-14.

19. Saving the 2nd Molar from the 3rd Is it Really the Guilt of the Tilt? / P. Yadav, P. J. Pruthi, R. R. Nawal [et al.] // Clin Diagn Res. — 2016. — 10. — P. $17-19$.

20. Черныш В. Ф. Совершенствование диагностики парадентальных кист челюстей // Междисциплинарный подход к диагностике, лечению и профилактике заболеваний тканей пародонта, у пациентов с сахарным диабетом: сб. мат. I междунар. науч.-практ. конф.— Санкт-Петербург, 2018.— С. 64-66. 
21. Ackermann G. The paradental cyst: a clinicopathologic study of 50 cases / G. Ackermann, M. A. Cohen, M. Altini // Oral Surg Oral Med Oral Pathol.— 1987 Sep.—64 (3). - P. 308312.

22. Vedtofte P. The inflammatory paradental cyst / P. Vedtofte, F. Praetorius // Oral Surg Oral Med Oral Pathol. — 1989 Aug. — 68(2). — P. 182188.

23. Histopathological evaluation of dental follicle associated with radiographically normal impacted mandibular third molars / N. Haidry, M. Singh, N. S. Mamatha [et al.].—D0l: 10.4103/ams.ams_215_18.//Ann Maxillofac Surg.— 2018. - 8.- P. 259-264.

24. Fowler C. B. The paradental cyst: a clinicopathologic study of six new cases and review of the literature / C. B. Fowler, R. B. Brannon // J Oral Maxillofac Surg. - 1989 Mar.-47(3).-P. 243248.

25. Characteristic MRI and cone beam CT findings in a case of paradental cysts arising in the bilateral retromolar regions of the mandible / A. S. Pinto, A. L. Costa, M. C. Pinto [et al.] // Journal of Oral and Maxillofacial Radiology. — 2016. — Vol. 4 (3). — P. 83.

26. Inflammatory paradental cyst (IPC) in the mandibular premolar region in children / Y. Morimoto, T. Tanaka, I. Nishida [et al.] // Oral Surg Oral Med Oral Pathol Oral Radiol Endod.-2004. - 97.- P. 286-293.

27. Mohan A. Inflammatory paradental cyst on the distobuccal aspect of an impacted mandibular third molar: A case report / A. Mohan, T. T. Sivakumar, A. P. Joseph [et al.] // Int. J. Case Rep. Images. — 2017.—Vol. 8 (9). — P. 592-596.

28. Paradental cyst of the first molar: A report of two cases / A. E. Borgonovo, S. Speroni, A. Fabbri, G. B. Grossi // J Indian Soc Pedod Prev Dent. — 2010 Apr-Jun. 28(2). - P. 116-120.

29. Анатомические причины развития ретенции третьих моляров на нижней челюсти / И. В. Гайворонский, В.Н. Николенко, А. К. Иорданишвили [и др.] // Курский научно-практический вестник «Человек и его здоровье».— 2015.— № 2. - C. 61-65.

30. Radiographic assessment of agenesis, impaction, and pararadicular radiolucencies in relation with third molar in Nashik City of Maharashtra / B.S. Ahire, A. R. Bhoosreddy, S. [et al.] // Journal of Dental and Allied Sciences. — 2016. - 5. - P. 3-9.

31. Диагностика и лечение затрудненного прорезывания нижних третьих моляров, осложненного перикоронаритом / Т.А. Пояркова, 0.В. Войковский, Н. С. Ковалева, Н. М. Стефанцов // Вестник Смоленской государственной медицинской академии. — 2014. — Т. 13.— № 3.— C. 107-110.

32. The predictivity of mandibular third molar position as a risk indicator for pericoronitis: A prospective study / P. Singh, P. Nath, S. Bindra [et al.]. — D0I: 10.4103/njms. NJMS_13_17. // Natl. J. Maxillofac. Surg. — 2018. — Vol. 9 (2)._P. 215-221. URL: https://www.ncbi.nlm.nih.gov/pmc/articles/PMC6251300/

33. The predictivity of mandibular third molar position as a risk indicator for pericoronitis: A prospective study / P. Singh, P. Nath, S. Bindra [et al.]. - D0I: 10.4103/njms. NJMS_13_17 // National Journal of Maxillofacial Surgery. — 2018 July-December. — Vol. 9. — Issue 2. - P. 215-221.

34. Murad N. Predesposing factors for the infection of mandibular third molars / N. Murad, Y. Khan, F. A. Qaiyum // Pak Oral Dent J. — 2013. — 33. — P. 253-256.

35. Factors associated with pericoronitis among subjects with impacted third molars teeth / S. A. Al-Hamdani, M. M. Al-Sened, K. T. Hussein, A. S. Al-Naaimi // MDJ. — 2011. - 8. — P. 193-201.

36. Impacted lower third molars and distal caries in the mandibular second molar. Is prophylactic removal of lower third molars justified? / J. Marques, MontserratM. Bosch, R. Figueiredo [et al.].—D0I: 10.4317/jced.53919.// J. Clin. Exp. Dent.—2017.—Vol. 9 (6).— P. e794-e798. URL: https://www.ncbi.nlm.nih.gov/pmc/ articles/PMC5474337/

37. Hashemipour M. A. Incidence of impacted mandibular and maxillary third molars: a radiographic study in a Southeast Iran population / M. A. Hashemipour, M. Tahmasbi-Arashlow, F. Fahimi-Hanzaei // Med Oral Patol Oral Cir Bucal. — 2013. - 18. — P. 140-145.

38. Effect of eruption status of the mandibular third molar on distal caries in the adjacent second molar / F. Kang, C. Huang, M. K. Sah, B. Jiang. - D0I: 10.1016/j. joms.2015.11.024. PMID: 26706495// J. Oral Maxillofac. Surg.— 2016. — Vol. 74. — P. 684-692.

39. Pathological sequelae of "neglected" impacted third molars / H. R. Stanley, M/ Alattar, W.K. [et al.] // Collett J Oral Pathol. — 1988. — 17. — P. 113-117.

40. Руденко А. Т. Патология прорезывания зубов мудрости / А. Т. Руденко.— Ленинград: Москва. — 1971.— 80 c.

41. Иорданишвили А. К. Анатомо-топометрические характеристики челюстей при прорезывании и ретенции зубов мудрости / А. К. Иорданишвили, Н. В. Коровин, А. А. Сериков. — D0I: 10.18481/2077-7566-2017-13-3-53-56// Проблемы стоматологии.— 2017.— Т. 13.— № 3.—C. 53-56.

42. Prevalence of odontogenic cysts and tumors associated with impacted third molars: a systematic review and meta-analysis / F.W. Mello, G. Melo, P.V. Kammer [et al.] // Journal of Cranio-Maxillofacial Surgery. — 2019._ - Vol. 47 (6)._ P. 996-1002.

43. Jones A. V. Range and demographics of odontogenic cysts diagnosed in a UK population over a 30year period. / A. V. Jones, G. T. Craig, C. D. Franklin // J Oral Pathol Med.- 2006 Sep.-35 (8).-P. 500507.

44. Paradental cysts: a role for food impaction in the pathogenesis. A review of cases from Northern Ireland / C. M. Colgan, J. Henry, S. S. Napier, C. G. Cowan // Br J Oral Maxillofac Surg. - 2002 Apr. - 40 (2). - P. 163168.

45. Reichart P. The development and fate of epithelial residues after completion of the human odontogenesis with special reference to the origins of epithelial odontogenic neoplasms, hamartomas and cysts / P. Reichart, H. P. Philipsen // Oral Biosci Med. - 2004. — 1(3). — P. 171179.

46. Prakash A.R Paradental cyst associated with supernumerary tooth fused with third molar: A rare case report / A.R Prakash, P.S. Reddy, M. Rajanikanth.- D DI: 10.4103/0973-029X.92991. Source: PubMed // Journal of Oral and Maxillofacial Pathology. — 2012 Apr — Vol. 16 — P. 131-133.

47. Kouhsoltani. M. Mandibular fracture associated with a dentigerous cyst: Report of a case and literature review/M. Kouhsoltani, A. H. Mesgarzadeh, M. J. MoradzadehKhiavi // Dent. Res. Dent. Clin. Dent. Prospects. — 2015._ 9. — P. 193-198.

48. Evaluation of the effects of the low-level laser therapy on swelling, pain, and trismus after removal of impacted lower third molar / H. Alan, Ü. Yolcu, M. Koparal [et al.]. — D DI: 10.1186/s13005-016-0121-1. // Head Face Med. — 2016. — Vol. 12. - 1. - P. 25.

49. Al-Hamed F. S. Clinical effects of platelet-rich fibrin (PRF) following surgical extraction of lower third molar / F. S. Al-Hamed, M.A.M. Tawfik, E. Abdelfadil //The Saudi Journal for Dental Research.— 2017.—Vol. 8 (1-2).—P. 19-25. URL: https://doi.org/10.1016/j.sjdr.2016.05.002 
50. Chrcanovic B. R. Gingival cyst of the adult, lateral periodontal cyst, and botryoid odontogenic cyst: an updated systematic review / B. R. Chrcanovic, R. S. Gomez. D0l: https://doi.org/10.1111/odi.12808// Oral diseases.—2019.—Vol. 25 (1). — P. 26-33.

51. The dentigerous cyst: two different treatment options illustrated by two cases / M. A. Bassetti, J. Kuttenberger, J. Novak, R. G. Bassetti // Swiss. Dent. J.— 2019. Vol. 129 (3). - P. 193-203.

52. Berezowski B. The early dentigerous cyst: a previously unrecognised lesion in the third molar region / B. Berezowski, V. Phillips, G. Cousin // Faculty Dental Journal.2018. - Vol. 9 (3)._P. 100-102. https://doi.org/10.1308/rcsfdj.2018.100

53. Antibiotic Prophylaxis on Third Molar Extraction: Systematic Review of Recent Data / Cervino G., Cicciù M., Biondi A. [et al.]. — D0l: 10.3390/antibiotics8020053. // Antibiotics (Basel). — 2019.—Vol.— 8 (2). — P. 53. URL: https://www.ncbi. nIm.nih.gov/pmc/articles/PMC6627726/

54. Dentigerous Cystic Changes in the Follicles Associated with Radiographically Normal Impacted MandibularThird Molars / A. Dongol, A. Sagtani, M. R. Jaisani [etal.]. doi.org/10.1155/2018/2645878.// International journal of dentistry.— 2018. — Vol. 2018.—URL: https://www.hindawi.com/journals/ijd/2018/2645878/

55. An Analysis of Dentigerous Cysts Developed around a Mandibular Third Molar by Panoramic Radiographs / M. Terauchi, S. Akiya, J. Kumagai [et al.]. — D0I: 10.3390/ dj7010013. // Dent. J. (Basel).— 2019.—Vol. 7 (1).—P. 13. URL: https://www.ncbi.nlm.nih.gov/pmc/articles/PMC6473924/

56. Слесарева 0. А. Современные методы диагностики и хирургического лечения фолликулярных кист челюстей у детей / 0. А. Слесарева, И. Ю. Карпова // Медицинский альманах. - 2019.— № 5-6 (61).—C. 101-103.

57. Three-dimensional position of mandibular third molars and its association with distal caries in mandibular second molars: a cone beam computed tomographic study / Y. Chen, J. Zheng, D. Li [et al.]. — D0I: 10.1007/s00784-020-03203-w. // Clin. Oral Investig. — 2020.— Vol. 10.— 1007/s00784-020-03203-w. PMID: 31938961

58. Radiographic signs of pathology determining removal of an impacted mandibular third molar assessed in a panoramic image or CBCT / L. H. Matzen, L. Schropp, R. Spin-Neto, A. Wenzel.— DOI: https://doi.org/10.1259/dmfr.20160330. // Dentomaxillofacial Radiology.— 2017.—Vol. 46 (1). 20160330. URL: https://www. birpublications.org/doi/full/10.1259/dmfr.20160330

59. Managing a Lateral Periodontal Cyst With Bone Graft: A Computed Tomography Assessment 18 Months Postoperatively / R. Livada, J. Shiloah, K. M. Anderson, W. R. Callahan // Compendium of continuing education in dentistry. - 2017. - Vol. 38 (1). - P. e5-e8.

60. Ярцева А. В. Оценка эффективности лучевой диагностики при планировании лечения одонтогенных и неодонтогенных кист челюстей / А. В. Ярцева, А. В. Гиенко // Современная парадигма естественных и технических наук: сб. науч. тр. по материалам Междунар. науч.-практ. конф., 29 апр. 2019 г.Белгород, 2019.- - С. 138-141.

61. Сравнительный анализ методов удаления ретинированных и дистопированных третьих нижних моляров / А. В. Трубина, Д. Д. Вахитова, А. А. Кузьмина, А. В. Бочарова // News of Science and Education. - 2019.— - T. 6. — № 5.— C. 86-89. URL: http://www.rusnauka.com/pdf/252458.pdf

62. Лобанова Н. И. Влияние воспаления и способа заполнения костной полости после удаления нижнего третьего моляра при его затрудненном прорезывании на длительность лечения / Н. И. Лобанова, В. П. Кирейчук, Е. С. Каган // Медицина в Кузбассе.— 2015.— № 3.— Т. 14.— C. $25-32$.

63. Bismuth subnitrate iodoform parafin paste used in the management of inflammatory follicular cyst - Report of two cases / A. Morawala, D. Shirol, Y. Chunawala [et al.]._D0I: 10.4103/JISPPD.JISPPD_328_16.//J. Indian Soc. Pedod. Prev. Dent.—2017._ Vol. 35 (3). — P. 269-274.

64. Postoperative effects on lower third molars of using mouthwashes with super-oxidized solution versus $0.2 \%$ chlorhexidine gel: A randomized double-blind trial / A. Coello-Gómez, S. Navarro-Suárez, J. M. Diosdado-Cano [et al.].— D0l: 10.4317/medoral.22622. // Med. Oral Patol. Oral Cir. Bucal.— 2018.—Vol. 23 (6).— P. 716-722. URL: https://www.ncbi.nlm.nih.gov/pmc/articles/PMC6260999/

65. Nahirniy Y.P. Research of the hole microflora after atypical removing of lower third molar in applying of osteoplastic material «KOLAPOL CP-3 LM» / Y.P. Nahirniy, R. V. Oshchypko, L.V. Piasetska // Clinical Dentistry. 2016. № 1. URL: https://doi.org/10.11603/2311-9624.2016.1.6151

66. Соловьев М. М. Показатель аутодезадаптации как один из критериев комплексной оценки состояния больного и эффективности применения нифлурила после удаления нижних третьих моляров / М. М. Соловьев, А. Clement // Оренбургский медицинский вестник.—2016. — T. IV. — № 3-1 (15). - C. 59-64.

67. Клинико-экспериментальное использование остеопластических средств при удалении ретенированных и дистопированных нижних третьих моляров / С. В. Сирак, А. А. Коробкеев, К. И. Сапунов, А. Д. Читанава // Саратовский научно-медицинский журнал. — 2010.— Т. 6. — № 1.— С. 136-139.

68. Лобанова Н. И. Использование плазмы, обогащенной тромбоцитами, при удалении ретенированного и дистопированного нижнего третьего моляра // Медицина в Кузбассе. - 2011.— № 3.— Т. 10.- С. 53-57.

69. Эффективность применения остеопластического материала «Стимул-ОСС» В сочетании с обогащённой тромбоцитами аутоплазмой при удалении дистопированных и ретинированных нижних третьих моляров / М. А. Токтомбаев, И. М. Юлдашев, А. Т. Борончиев, Ж. В. Ашим0B. - D0I: 10.25005/2074-05812017-19-4-513-519// Вестник Авиценны. — 2017. — Том 19.— № 4.- С. 513-519.

70. Применение пьезохирургии при лечении парадентальных кист / Т. Л. Маругина, В. В. Кан, В. В. Федотов, Е. С. Загородних // Современные исследования социальных проблем (электронный научный журнал).— № 12(20).— 2012.— URL: https: // www.sisp.nkras.ru 